\title{
Extraction of Stable Points from Fingerprint Images Using Zone Could-be-in Theorem
}

\author{
Xuchu Wang ${ }^{1}$, Jianwei $\mathrm{Li}^{1}$, Yanmin $\mathrm{Niu}^{2}$, Weimin Chen ${ }^{1}$, and Wei Wang ${ }^{1}$ \\ ${ }^{1}$ Key Lab on Opto-Electronic Technique of State Education Ministry, \\ Chongqing University, 400044, Chongqing, P.R. China \\ Seadrift@sina.com, jwli@cqu.edu.cn \\ ${ }^{2}$ College of Physics and Information Techniques, Chongqing Normal University, \\ 400047, Chongqing, P.R. China
}

\begin{abstract}
This paper presents a novel zone Could-be-in theorem, and applies it to interpret and extract singular points (cores and deltas) and estimate directions of cores in a fingerprint image. Where singular points are regarded as stable points (attracting points and rejecting points just according to their clockwise or anticlockwise rotation), and pattern zones are stable zones. Experimental results validate the theorem. The corresponding algorithm is compared with popular Poincaré index algorithm under two new indices: reliability index (RI) and accuracy cost (AC) in FVC2004 datasets. The proposed algorithm are higher $36.49 \%$ in average RI, less 2.47 in average AC, and the advantage is more remarkable with the decrease of block size.
\end{abstract}

\section{Introduction}

Singular points (SPs) are global features in fingerprint images and play an important role in fingerprint identification/authentication [1]. Henry defined two early types of singular points, where a core is the topmost point of the innermost curving ridge and a delta is the center of triangular regions where three different direction flows meet [2]. Since the directional field around SPs is discontinuous, many approaches intended to solve the problem by orientation distribution [3][4][5][6][7][8]. Now the popular and elegant detecting method is Poincaré index based approach [9][10], and point orientation is often replaced by block orientation due to efficiency. Ref. [9] made some useful improvements to quicken detection. Nevertheless, little attention was focused on the definition of SPs and the direction estimation of core points in previous research on this topic.

SP is more regarded as a region than a point and it can be represented by a barycenter of the region. Different methods lead to different positions while they are situated in a similar region, so the reliability must be considered firstly and then be the accuracy. A limitation of Poincare index method is the contradiction of reliability and accuracy. Another limitation is that when the noise is heavy, more pseudo SPs will be gotten or right points will be omitted due to increasing false orientations [1][4], so [9] proposed to refuse pseudo points by iterative smoothing method and which reduced accuracy. The third is the method can't estimate directions of core 
points. Hence, it is an especially challengeable problem to improve the reliability of SPs without extra expenditure.

In this paper, we present new idea to interpret and detect SPs. The fingerprint orientations are interpreted by some original definitions in a dynamic viewpoint, where SPs are regarded as stable points surrounded by shortest stable boundary. When the stable points are rotating clockwise, they are assumed to get an ability of attracting ridges and other stable points, we call them as attracting points. Similarly, when they are rotating anticlockwise they are rejecting points with rejecting ability. The pattern zones around the stable points are regarded as stable zones. We propose a zone Could-be-in theorem to extract the stable points and estimate the directions of core points simultaneously by analyzing the property of the shortest stable boundary. (All of them are included in the fingerprint growth model proposed by the author.) We also define reliability index (RI) and accuracy cost (AC) to evaluate different performances of extraction algorithms. Experimental results show that our algorithm is higher $36.49 \%$ in $\mathrm{AI}$ and less 2.47 in AC than Poincaré index algorithm. When the block size is decreased, the advantage of our algorithm is more remarkable.

\section{Zone Could-be-in Theorem}

According some statistical analysis about orientations of ridges in fingerprint images, some results in nonlinear dynamic system, we present some definitions as follows:

Discrete orientation field $\boldsymbol{O}$ : A support set in 2-dimension plane composed by a serial of directional particles in square meshes. The term is written as $O=\left\{K_{i} \mid \theta_{i} \in[0, \pi), i \in Z\right\}$, we use orientation to describe directionality in images for distinction, so $\theta_{i}$ is the orientation of particle $K_{i}$;

Could-be-in: If the orientation of $K_{1}, K_{2}, K_{3}$ in $\boldsymbol{O}$ can be described as $\left|\theta_{1}-\theta_{2}\right|>\theta_{3}$,we call K3 is Could-be-in to K1,K2. Suppose $\theta_{1} \leq \theta_{2}$, the term is written as $K_{3} \mapsto\left\langle\hat{K}_{1}, \hat{K}_{2}\right\rangle \cdot$

Zone Could-be-in: There are $K_{p}$ and a sequence of $\left\{K_{i} \mid i=0,1, \ldots L-1\right\}$ in $\boldsymbol{O}$, $K_{p} \notin\{K i\}$, if the term $K_{p} \mapsto\left\langle K_{s}, K_{(s+1)} \bmod L\right\rangle ; s \in\{0,1, \ldots L-1\}$ is true, then we regard that the loop $L\left\{K_{i} \mid i=0,1, \ldots L-1\right\}$ composed by $\left\{K_{i} \mid i=0,1, \ldots L-1\right\}$ is Zone Could-be-in to $K_{p}$. The term is written as $K_{p} \mapsto \operatorname{LOOP}\left\langle K_{1}, K_{2}, \ldots, \hat{K}_{s}, \hat{K}_{(s+1)} \bmod L_{L} \ldots K_{L-1}\right\rangle$. The “^” symbolizes the entrance position of $K_{p}$. We can get Entrance Times $\mathrm{N}$ according to the number of " $\wedge$ ". If $\mathrm{N}$ is equal to $\mathrm{L}$, the zone is Could-be-in to $K_{p}$ everywhere. If $\mathrm{N}$ is equal to 2 , the zone is Could-be-1-in to $K_{p}$. If $\mathrm{N}$ is less than 2 , the Zone is not Could-be-in to $K_{p}$. Apparently Could-be-in is a special case of Zone Could-be-in.

Monotone zone Could-be-in: There are $K_{p}$ and $\left\{K_{i} \mid i=0,1, \ldots L-1\right\}$ where $K_{p} \notin\{K i\}$, $K_{p} \mapsto L O O P\left\langle K_{1}, K_{2}, \ldots, \hat{K}_{s}, \hat{K}_{(s+1)} \bmod L \ldots K_{L-1}\right\rangle$, if $\left\{K_{i}\right\}$ is monotone and $L\left\{K_{i}\right\}$ is 
Monotone zone Could-be-in to $K_{p}$, the term is written as $K_{p} \mapsto \overrightarrow{\operatorname{LOOP}}\left\langle K_{0}, K_{1}, \ldots, \hat{K}_{s}, \hat{K}_{(s+1)} \bmod L \ldots K_{L-1}\right\rangle$. If only one $s$ can let the term true, we call $L\left\{K_{i}\right\}$ is Monotone Zone Could-be-1-in to $K_{p}$, so $K_{p} \mapsto \overrightarrow{\operatorname{LOOP}}\left\langle\hat{K}_{0}, K_{1}, \ldots, \hat{K}_{L-1}\right\rangle$.

Stable Zone: There are $K_{p}$ and $\left\{K_{i} \mid i=0,1, \ldots L-1\right\}$ where $K_{p} \notin\{K i\}$, if $K_{p}$ is in loop $L\left\{K_{i} \mid i=0,1, \ldots L-1\right\} \quad$ and term $\quad K_{p} \mapsto \overrightarrow{\operatorname{LOOP}}\left\langle\hat{K}_{0}, K_{1}, \ldots, \hat{K}_{L-1}\right\rangle \quad$ is true, then $L\left\{K_{i} \mid i=0,1, \ldots L-1\right\}$ can be regarded as a gradual stable zone to $K_{p}$. The shortest one of all $L\left\{K_{i}\right\}$ is regarded as the stable zone to $K_{p}$. It is reasonable to call $\left\{K_{i}\right\}$ as the boundary sequence of the zone. If the length of $\left\{K_{i}\right\}$ is in range $[4,8]$, we further call it the shortest stable boundary to $K_{p}$ and $K_{p}$ is a stable point.

Term zone Could-be-in describes relationship of an orientation and a sequence of orientation. It can be interpreted from two aspects, one is that the entrance orientation is accord with the orientation loop and it can be a part of the zone surrounded by orientation loop, the other is that there is a directional particle which can attract or reject the orientation loop. By the mutual function, both of them get a stable status. That's the reason we call them "stable point" and "stable boundary". Like some handedness phenomenon in particle physics field, we assume the attracting or rejecting ability is the property of a particle and determined just by rotating direction of the particle. As Fig.1 depicts, the shortest stable boundary is convex or concave in order to get a harmony It's apparent that the orientation loop around a stable point must satisfy some conditions to get a kind of harmony, which will be discussed by the following theorem.

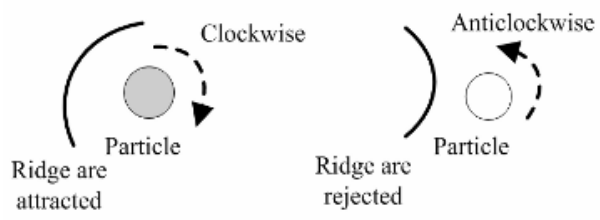

Fig. 1. Attracting or rejecting ability of a particle

Theorem. If a sequence is monotone zone Could-be-1-in to a directional particle, the entrance position must be between the extremums of the sequence.

Proof. Let $K_{p}$ be the directional particle and $\left\{K_{i} \mid i=0,1, \ldots L-1\right\}$ be the sequence, since $\left\{K_{i}\right\}$ is monotone, it can be arranged as a loop called $L\left\{K_{i}\right\}$ in which the maximum and minimum is neighbor ( $\max$, min represent their positions in same period of the loop and $\theta_{\max }, \theta_{\min }$ are the corresponding orientations). Assume the entrance position of $K_{p}$ is mid (mid is not equal to max or min), hence MIN \{max, $\min \}<\operatorname{mid}<\operatorname{MAX}\{\max , \min \}, \operatorname{MAX}$ and MIN are getting extremums operation, 
respectively. Since $\theta_{\min }<\theta_{\text {mid }}<\theta_{\max }$ and we can disconnect $L\left\{K_{i}\right\}$ at mid position and get sequence $\left\{K_{i}^{\prime}\right\}$, which is descending from mid to min, and ascending from max to mid, so $\left\{K_{i}^{\prime}\right\}$ is not a monotone sequence. This means mid position cannot be gotten, the one and only entrance position is between min and max unless the orientations of all directional particle are equal, which contradicts the monotony of the sequence.

Q.E.D.

Further suppose the length of $L\left\{K_{i}\right\}$ is $\mathrm{L}$, disconnect it to a monotone ascending sequence $\left\{K_{i}\right\}$, let $\Delta \theta_{i}=\theta_{(i+1) \bmod L}-\theta_{i} ; i=0,1 \ldots L-1$, then

$$
\begin{gathered}
\theta_{p}>\Delta \theta_{i}>0 ; \quad i=0,1 \ldots L-2 . \\
\theta_{p}<\Delta \theta_{L-1}
\end{gathered}
$$

The theorem, especially the two inequations above, qualifies the relationships of $K_{p},\left\{K_{i}\right\}, L\left\{K_{i}\right\}$ and supplies a criterion of existence of stable boundary. Note that there are many methods to detect the existence of a stable boundary and we just provide one way here, for some case, Poincaré index is a detecting method, too. When $\mathrm{L}$ is $8,\left\{K_{i}\right\}$ becomes an eight-direction stable boundary. It also provides a way to extract stable points through detecting the eight-direction stable boundary. By the way, the entrance position is a clue to estimate the direction of a core point in a fingerprint image and we will discuss it in the following section.

\section{Extraction Methodology}

As a description of orientation changing rule of discrete orientation, zone Could-be-in theorem provides some perspectives to detect some special zone and zone distribution. It can be used to extract fingerprint SPs and the algorithm procedure is as follows:

Step.1 - Segmenting background by variance threshold method.

Step.2 - Building discrete orientation field. Divide fingerprint image $M$ into blocks of size $W_{i} \times W_{i}(16 \times 16)$ and use the least square method [11] to estimate a directional image. The result is regarded as a discrete orientation field $O_{\text {all }}$ of the whole image. If the input is a part of fingerprint image, the corresponding $O$ is $O_{\text {part }} i$.

Step.3-Detecting stable zones by zone Could-be-in theorem. Locate the 8-connected zones of the stable zones and divide them into $O_{\text {core }}$ and $O_{\text {delta }}$.

Step.4-Overlapping and location. Map the regions of $M$ by $O_{\text {core }}, O_{\text {delta }}$ and decrease the block size $W_{i} \times W_{i}$ as $12 \times 12,8 \times 8$, return to step 2,3 to get $O_{\text {part_1 } 1} ; O_{\text {part_2 } 2} ; \ldots$

Step.5-Break out when $W_{i}$ is less than a presetting threshold.

The direction of a core point can provide very useful information for fingerprint classification and fingerprint matching even though it is not very 
accurate. A little literature discussed this topic such as [6], and the method is computationally expensive. While in zone Could-be-in theorem, this problem can be easily solved. Firstly, we formulate the entrance position $i$ and directional range $L i$ :

$$
L_{i}=\left[\lfloor(i+1) / 2\rfloor \times \pi / 2+(-1)^{i} \beta,(1+\lfloor(i+1) / 2\rfloor) \times \pi / 2+(-1)^{i} \beta\right) ; i=0,1, \ldots 7
$$

where $\beta=\operatorname{arctg}(1 / 2)$ and the length of every range is $\pi / 2,\lfloor\bullet\rfloor$ means floor integer operation. Note that when i is 6 or 7 , the range is composed by two sub-ranges. Secondly, let $\theta_{p}$ be the orientation of a stable point and $\theta_{i}, \theta_{(i+1) \bmod 8}$ be the extremums of its eight-direction shortest stable boundary, considering they are orientations in range $[0, \pi)$, we map an orientation to the direction range $L i$ by a function $f\left(\theta_{i}\right)$.Lastly, we consider three elements dominate a core point direction $\beta_{\text {core }}$ together:

$$
\begin{gathered}
f\left(\theta_{i}\right)= \begin{cases}\theta_{i}+\pi ; & \theta_{i}+\pi \in L_{i} \\
\theta_{i}+\pi / 2 ; & \theta_{i}+\pi / 2 \in L_{i} \\
\theta_{i}-\pi / 2 ; & \theta_{i}-\pi / 2 \in L_{i} \\
\theta_{i} ; & \theta_{i} \in L_{i}\end{cases} \\
\beta_{\text {core }}=\lambda_{1} f\left(\theta_{i}\right)+\lambda_{2} f\left(\theta_{(i+1) \bmod 8}\right)+\lambda_{3} f\left(\theta_{p}\right)
\end{gathered}
$$

where $\mathrm{i}$ is entrance position, $\lambda_{1}, \lambda_{2}, \lambda_{3}$ are weighted coefficients $(0.3,0.3,0.4$ empirically).

\section{Experimental Results}

Some detecting results and the comparision with popular Poincaré index algorithm under different block sizes are shown in Fig.2. In order to emphasis locations, some directions of the core points in our algorithm are omitted.

Apparently the locations of singular points in both methods are similar and overlapped in some portions. We define two indices: reliability index $(R I)$ and accuracy cost $(A C)$ to evaluate the performance of different algorithms:

$$
R I=R Z / T Z \times 100 \% ; A C=R N / T N
$$

where $T Z$ is the number of total zones detected according to 8-connectness, $R Z$ is the number of right zones determined by human experts, $T N$ is the total number of detected SPs, and $R N$ is the number of SPs in right zones. The ideal performance of a singular point extraction algorithm is that $R I$ is near to $100 \%$ and $A C$ is near to 1 . Table 1 reports the comparison matrix about average value of every image in FVC2004 and induces some conclusions: (i) average $R I$ and $A C$ of Alg.ZC are higher $36.49 \%$, less 2.47 than those of Alg.P. (ii) advantage of Alg.ZC is more remarkable with decrease of block size. 


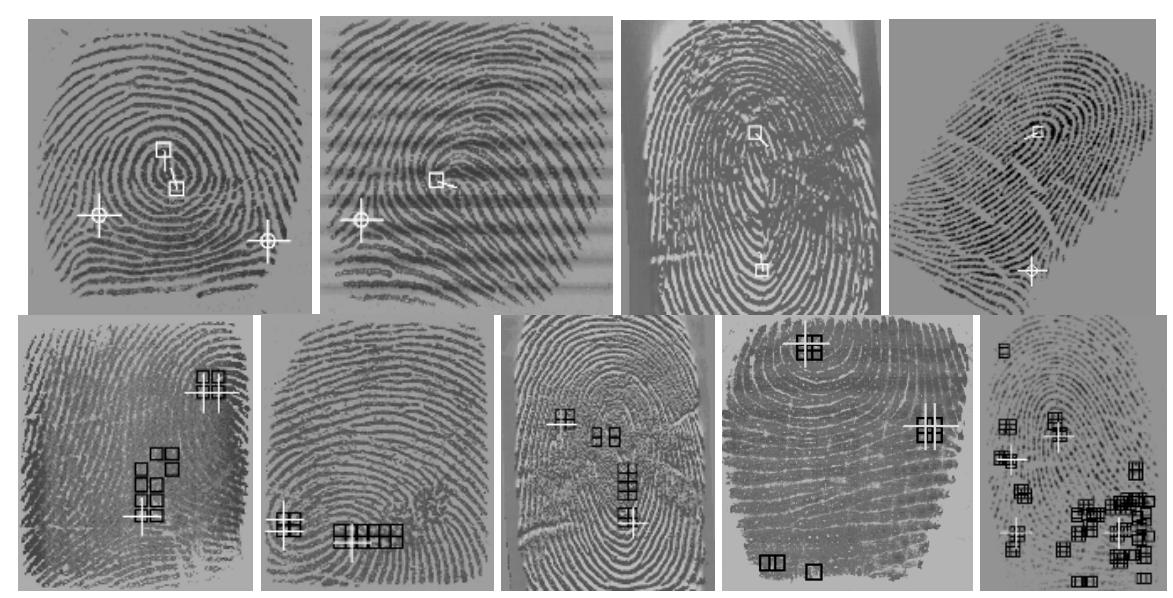

Fig. 2. Up row is some results of Alg.ZC, and low row is comparion by Alg.ZC ("+") and Alg.P (“ $\square$ ”) under different block size

Table 1. Extraction matrix by zone Could-be-in algorithm (Alg.ZC) and by Poincaré index algorithm (Alg.P) with block sizes 16,12 and 8 in FVC2004 four datasets

\begin{tabular}{|c|c|c|c|c|c|c|c|}
\hline$D B$ & Alg. & $T Z$ & $R Z$ & $T N$ & $R N$ & $R I(\%)$ & $A C$ \\
\hline \multirow{2}{*}{1} & Alg.ZC & $1.41,1.43,1.49$ & 1.35 & $1.62,1.67,1.72$ & $1.46,1.52,1.64$ & $95.74,94.40,90.60$ & $1.08,1.13,1.21$ \\
\hline & Alg.P & $2.29,2.46,3.02$ & 1.35 & $7.87,8.03,10.94$ & $5.28,5.33,5.37$ & $58.95,54.88,44.70$ & $3.91,3.95,3.98$ \\
\hline \multirow[b]{2}{*}{2} & Alg.ZC & $1.56,1.61,1.73$ & 1.25 & $1.87,1.95,2.03$ & $1.52,1.78,1.91$ & $80.13,83.85,72.25$ & $1.22,1.42,1.53$ \\
\hline & Alg.P & $2.89,3.90,5.18$ & 1.25 & $\begin{array}{l}10.04,12.06 \\
8.11\end{array}$ & $4.93,4.95,4.98$ & $43.25,32.05,24.13$ & $3.94,3.96,3.98$ \\
\hline \multirow[b]{2}{*}{3} & Alg.ZC & $2.37,2.38,2.41$ & 2.24 & 4.17,4.21,4.40 & $4.06,4.13,4.35$ & $94.51,94.12,92.95$ & $1.81,1.84,1.94$ \\
\hline & Alg.P & $2.55,2.76,3.47$ & 2.24 & $\begin{array}{l}9.88,10.10 \\
13.22\end{array}$ & $8.69,8.71,8.72$ & $87.84,81.16,64.55$ & $3.88,3.89,3.89$ \\
\hline \multirow{2}{*}{4} & Alg.ZC & $1.70,1.77,1.85$ & 1.61 & $2.07,2.14,2.34$ & $1.92,2.03,2.28$ & $94.70,90.96,87.03$ & $1.19,1.26,1.42$ \\
\hline & Alg.P & $2.98,3.36,4.04$ & 1.61 & $8.23,8.92,13.26$ & $6.06,6.09,6.11$ & $54.03,47.92,39.85$ & $3.76,3.78,3.80$ \\
\hline \multirow{2}{*}{ Avg } & Alg.ZC & 1.81 & 1.61 & 2.51 & 2.39 & 89.27 & 1.42 \\
\hline & Alg.P & 3.24 & 1.61 & 10.89 & 6.27 & 52.78 & 3.89 \\
\hline
\end{tabular}

\section{Conclusions and Future Work}

The contribution of this paper lies in three points:

(i) Define the singular points as stable points (attracting points and rejecting points just by their rotation) and pattern zones are stable zones from a new viewpoint.

(ii) Propose some innovative definitions and a theorem called zone Could-be-in theorem to extract the stable points and their directions. 
(iii) Define two indices: reliability index (RI) and accuracy cost (AC) to evaluate the performance of different extraction algorithms. The average RI, AC of our proposed algorithm are higher $36.49 \%$, less 2.47 than those of Poincaré index based algorithm in four FVC2004 datasets, the advantages are more remarkable when block size decreases.

In further research we will apply these ideas to enhance and classify fingerprints.

\section{References}

1. D. Maltoni, D. Maio, A. K. Jain, S. Prabhakar: Handbook of Fingerprint Recognition. Springer, New York, (2003)96-99

2. E. R. Herny: Classification and Uses of Finger Prints. George Routledge \& Sons, London, 1900

3. V.S.Srinivasan, N.N.Murthy: Detection of singular points in fingerprint images. PR, 25(1992)139-153

4. Marius Ticu, Pauli Kuosmanen: A multiresolution method for singular points detection in fingerprint images, Proc. 1999 IEEE ISCS, 4(1999)183-186

5. X. Wang, J. Li, and Y. Niu: Fingerprint Classification Based on Curvature Sampling and RBF Neural Networks, Lecture Notes in Computer Science, 3497(2005)171-176

6. Asker M. Bazen and Sabih H. Gerez: Systematic methods for the computation of the directional fields and dingular points of fingerprints. IEEE Trans. PAMI, 24(2002)905919, 2002

7. Maio D, Maltoni D: A structural approach to fingerprint classification. Proc. 13th ICPR, (1996)578-585

8. M. Kawagoe, A. Tojo: Fingerprint pattern classification. PR, 17(1984)295-303

9. K. Karu, A. K Jain: Fingerprint classification, PR, 29(1996)389-404

10. Nojun Kwak, Chong-Ho Choi: Input feature selection by mutual information based on Parzen window. IEEE Trans. PAMI,24(2002)1667-1771

11. L.Hong, Y.Wan, and A. Jain: Fingerprint image enhancement: algorithm and performance evaluation. IEEE Trans. PAMI, 20(1998)777-789 